\title{
Neonatal and maternal platelet cytosolic calcium in normotensive and hypertensive pregnancies
}

\author{
Mark D Kilby, Fiona Broughton Pipkin, E Malcolm Symonds
}

\begin{abstract}
A prospective study investigated platelet cytosolic calcium in non-pregnant volunteers $(n=30)$ and samples from the umbilical veins of babies from both normotensive $(n=18)$ and hypertensive $(n=15)$ primigravidae, and their mothers. There was no significant difference between the neonatal umbilical venous platelet cytosolic calcium concentration $\left(\mathrm{p}\left[\mathrm{Ca}^{2+}\right] \mathrm{i}\right)$ in babies born to normotensive primigravidae or to those whose pregnancies were complicated by gestational hypertension (88.9 (SE) 2.5 ) in normotensive primagravidae, $80.6(2.8)$ in pregnancy induced hypertension without proteinuria, and $89.3(3.2) \mathrm{nmol} / \mathrm{l}$ in pre-eclampsia. There was also no significant difference in the $\mathrm{p}\left[\mathrm{Ca}^{2+}\right] \mathrm{i}$ from the umbilical veins of the pregnancies studied and those of non-pregnant female volunteers in the follicular phase of their menstrual cycle. This was despite a gradual and significant rise in $\mathbf{p}\left[\mathrm{Ca}^{2+}\right] \mathbf{i}$ with increasing severity of disease
\end{abstract} in the mothers of the babies studied (119.9 (4.1) in normotensive primagravidae, $130.8(7 \cdot 3)$ in pregnancy induced hypertension without proteinuria, and $148 \cdot 2$ (4.5) $\mathrm{nmol} / \mathrm{l}$ in pre-eclampsia). The mean maternal $\mathrm{p}\left[\mathrm{Ca}^{2+}\right] \mathrm{i}$ in the three samples returned to concentrations comparable with those in non-pregnant subjects by 12 weeks after birth.

These data demonstrate no significant difference between the mean $\mathrm{p}\left[\mathrm{Ca}^{2+}\right] \mathrm{i}$ in non-pregnant women and those obtained from the umbilical venous blood of normotensive or hypertensive primigravidae. They suggest that the functional hypoactivity of neonatal platelets is probably not secondary to a decrease in basal $\mathrm{p}\left[\mathrm{Ca}^{2+}\right] \mathrm{i}$. They also suggest that the progressively raised $\mathrm{p}\left[\mathrm{Ca}^{2+}\right] \mathbf{i}$ in normal and hypertensive pregnancies might be due to a pregnancy specific factor that does not cross the placenta.

(Arch Dis Child 1994; 71: F6-F10)

Platelets are anucleate cellular fragments that circulate in blood. They have a prime haemostatic function in that they undergo receptor mediated aggregation and exocytosis in response to endothelial injury. ${ }^{1}$ There is evidence that platelet function is at least, in part, controlled by cytosolic free calcium concentrations. ${ }^{2}$ Fetal and neonatal platelets have a tendency towards, 'hyporeactivity' compared with adults when tested ex vivo. ${ }^{3-6}$ To our knowledge, the cytosolic free calcium concentration in the platelets of these infants has not been measured. Thus the hyporeactivity might reflect either a lowered basal value or a diminished rise of calcium 'transient' in response to an agonist:receptor interaction. The purpose of this study was to determine whether platelet cytosolic calcium concentration $\left(\mathrm{p}\left[\mathrm{Ca}^{2+}\right] \mathrm{i}\right)$ was indeed lower in platelets from the umbilical cord blood of newborn babies. As women who suffer hypertensive disease of pregnancy have raised $\mathrm{p}\left[\mathrm{Ca}^{2+}\right] \mathrm{i},{ }^{7}$ we also measured this in the platelets of their neonates to determine whether a maternal factor(s) might be influencing neonatal platelet function.

\section{Patients and methods}

SUBJECTS

The study was approved by the hospital's committee on ethics and all mothers gave informed consent. All subjects were in their first pregnancy and the diagnosis of gestational hypertension was made on the antenatal ward at Queen's Medical Centre, Nottingham, at least 24 hours before the onset of labour.

The control pregnant subjects were 18 normotensive primiparous women, all in spontaneous labour, who had attended the antenatal clinic as part of an ongoing prospective study. All subjects were also investigated at 12 weeks after birth.

Data for comparison was obtained from 15 primiparous subjects whose pregnancies were complicated by gestational hypertension. No subjects were taking antihypertensive treatment. Pregnancy induced hypertension was defined as a blood pressure of at least 140/90 $\mathrm{mm} \mathrm{Hg}$ on two occasions 24 hours apart, and was verified by a second observer. Six women had hypertension alone, while a further nine had 'significant' proteinuria (at least $0.5 \mathrm{~g} / 24$ hours; salicylsulphonic acid turbidity method) and were thus defined as having pre-eclampsia. These subjects were matched for age and weight. Some demographic data are demonstrated in table 1 . Venous samples were taken 
Table 1 Demographic data of patients studied; values are mean (SE)

\begin{tabular}{|c|c|c|c|c|}
\hline & $\begin{array}{l}\text { Non-pregnant } \\
\text { volunteers } \\
(n=30)\end{array}$ & $\begin{array}{l}\text { Normotensive } \\
\text { primagravidae } \\
(n=18)\end{array}$ & $\begin{array}{l}\text { Pregnancy induced } \\
\text { hypertension } \\
\text { without } \\
\text { proteinuria } \\
(n=6)\end{array}$ & $\begin{array}{l}\text { Pre-eclampsia } \\
(n=9)\end{array}$ \\
\hline $\begin{array}{l}\text { Age (years) } \\
\text { Maternal weight }(\mathrm{kg})\end{array}$ & $\begin{array}{l}28.3(0.7) \\
64.8(1.9)\end{array}$ & $\begin{array}{l}26 \cdot 3(1 \cdot 2) \\
78 \cdot 8(2 \cdot 1)\end{array}$ & $\begin{array}{l}22 \cdot 0(1.4) \\
92 \cdot 3(7 \cdot 0)^{\star}\end{array}$ & $\begin{array}{l}25 \cdot 2(1 \cdot 2) \\
82 \cdot 7(4 \cdot 2)\end{array}$ \\
\hline \multicolumn{5}{|l|}{ Blood pressure $(\mathrm{mm} \mathrm{Hg})$} \\
\hline Diastolic & $\begin{array}{r}108.4(1.0) \\
64.7(1.2)\end{array}$ & $\begin{array}{r}112 \cdot 4(1 \cdot 9) \\
71.6(1.7)\end{array}$ & $\begin{array}{r}147 \cdot 8(2 \cdot 4)^{\star \star \lambda} \\
94 \cdot 8(3 \cdot 3)^{\star \star \star}\end{array}$ & $\begin{array}{r}150.7(0.8)^{\star \star \star} \\
94.8(2 \cdot 2)^{\star \star \star}\end{array}$ \\
\hline Gestation (weeks) & - & $40 \cdot 1(0 \cdot 5)$ & $38 \cdot 6(1 \cdot 1)$ & $36 \cdot 2(1 \cdot 3)^{\star}$ \\
\hline Birth weight (g) & - & $3400(100)$ & $2900(200)$ & $2650(300)^{\star \star}$ \\
\hline $\operatorname{Sex}(M: F)$ & - & $1: 1 \cdot 8$ & $1 \cdot 5: 1$ & $1 \cdot 8: 1$ \\
\hline IUGR ( $<10$ th centile $)$ & - & 0 & 1 & 4 \\
\hline
\end{tabular}

${ }^{\star} p<0.05 ;{ }^{\star \star} p<0.01 ;{ }^{\star \star \star} \mathrm{p}<0.0001$. IUGR =intrauterine growth retardation.

From the data shown it should be noted that the study samples were not statistically different in terms of age, although the non-proteinuric group were somewhat younger $(p=0.09)$. The mean maternal weight was significantly heavier in the group with pregnancy induced hypertension without proteinuria compared with the other samples $(p<0 \cdot 05)$. The mean systemic blood pressures taken in the late intrapartum period, at the time of study are demonstrated. The subjects with gestational hypertension by definition have arterial blood pressures raised compared with the normotensive primigravidae, the diagnosis having been made in the antenatal period. There was a significant difference in the mean gestation of the pregnancy at delivery between the sample groups. The mean gestation was lowest in the mothers with pre-eclampsia compared with the normotensive primagravidae $(36 \cdot 2(1 \cdot 3) v 40 \cdot 1(0 \cdot 5)$
weeks; $p<0.05)$. This was most probably a reflection of the increased induction rate in weeks; $\mathrm{p}<0.05)$. This was most probably a refle
those subjects with gestational hypertension.

from the umbilical vein, after the birth of the baby, but before placental separation. Simultaneously, a venous sample was taken from the mother.

The arterial blood pressure was taken with the mother in a supine position with a slight left sided tilt. All blood pressures were measured by the same observer and were measured in the right arm at the level of the right atrium, using a Hawksley random zero sphygmomanometer. Korotkoff phase IV was taken as an estimate of diastolic blood pressure following recommendations for the most accurate indirect determination in pregnancy. ${ }^{8}$ Two measurements were taken five minutes apart and the second reading recorded. Pregnant women who had had a blood pressure of $140 / 90 \mathrm{~mm} \mathrm{Hg}$ or greater at less than 20 weeks' gestation were excluded and all patients recruited were normotensive at their antenatal booking visit and again at 12 weeks after birth.

None of these women were taking any medications, apart from haematinic preparations and none were known to be suffering from renal, cardiovascular, or metabolic disease.

So as to make a valid comparison between

Table 2 Paired differences in $\mathrm{p}\left[\mathrm{Ca}^{2+}\right] \mathrm{i}$ in umbilical and maternal venous blood in the different groups studied (both antenatally and 12 weeks after birth)

\begin{tabular}{lccl}
\hline & Mean difference & $S E$ & p Value \\
\hline Normotensive primagravidae & & & \\
$\quad$ Maternal-umbilical & 31.06 & 3.84 & 0.0001 \\
Umbilical-postnatal & -4.0 & 2.94 & 0.19 \\
Pregnancy induced hypertension & 50.16 & 7.05 & 0.0001 \\
$\quad$ Maternal-umbilical & -11.17 & 7.03 & 0.17 \\
Umbilical-postnatal & 58.88 & 6.2 & 0.0001 \\
Pre-eclampsia & -4.0 & 2.8 & 0.19 \\
Maternal-umbilical & Umbilical-postnatal & & \\
\hline
\end{tabular}

Paired analysis of differences demonstrated a significant difference between the cytosolic calcium concentrations in the normotensive primagravidae and their babies (95th confidence interval 22.94 to $39 \cdot 16 ; \mathrm{p}<0.0001$ ). This was also noted in the gestational hypertensive groups, the mean difference being greatest with the most severe maternal disease. No significant difference was noted between the umbilical cord $\mathrm{p}\left[\mathrm{Ca}^{2+}\right] \mathrm{i}$ in either babies born to normotensive women or those born to women with pregnancy induced hypertension without normotensive women or those born to women with pregnancy induced hypertension without
proteinuria or with pre-eclampsia (ANOVA, $p>0 \cdot 1$ ). Also, there was no significant difference between paired differences in umbilical cord $\mathrm{p}\left[\mathrm{Ca}^{2+}\right]$ i and those 12 weeks postnatally in any of the groups studied. neonatal (umbilical) and adult $\mathrm{p}\left[\mathrm{Ca}^{2+}\right] \mathrm{i}$, a control group of 30 non-pregnant, nulliparous subjects were also studied to exclude the influence of pregnancy per se on platelet function.

\section{STUDY PROTOCOL}

Subjects underwent venesection from the antecubital fossa (in the mothers) or umbilical cord using a 19 gauge needle before placental separation. Samples were drawn for the measurement of total serum calcium concentration (Kodak Ektachem model 70 XR analyser) and albumin (colour complexion method). A full blood count, including platelet count, was made (Coulter S, Plus 4 counter method) and venous blood was taken for estimation of $\mathrm{p}\left[\mathrm{Ca}^{2+}\right] \mathrm{i}$.

\section{MEASUREMENT OF $\mathrm{p}\left[\mathrm{Ca}^{2+}\right] \mathrm{i}$}

These were determined using the fluorescent indicator, fura-2/AM using a modification of the method ${ }^{9}$ fully described in our previous paper. ${ }^{10}$ The intracellular fura-2 concentration was between $15-80 \mathrm{mmol} / 1$ of cell water. The final cell suspension was adjusted to a concentration of $1 \times 10^{8}$ platelets $/ \mathrm{ml}(5 \cdot 5 \%)$. Fluorescence measurements were performed on a Perkin-Elmer LS 3B fluorescence spectrophotometer at excitation and emission wavelength settings of $339 \mathrm{~nm}$ and $495 \mathrm{~nm}$ respectively. The measurement was made in triplicate for each patient. The intra-assay and 'day to day' coefficients of variation were $10 \cdot 4 \%$ and $7 \cdot 8 \%$ respectively. The time between drawing blood and the end of the estimation was approximately two hours.

\section{STATISTICS}

The data on examination were found to be normally distributed with no significant degree of skewness. All data are thus represented as mean (SE) unless otherwise stated. Analysis of the data was carried out after consultation with the University of Nottingham Statistical Adviser (Mr P H Riley) using the Statistical Package for the Social Sciences (SPSS, 6.0 for Windows). ${ }^{11}$ One way analysis of variance (ANOVA) was carried out using Scheffé's test for group differences. Where only two group means were to be compared a $t$ test was used. The mean differences and their standard errors are analysed where comparisons of paired data are shown (table 2).

\section{Results}

(A) DEMOGRAPHIC DATA

The demographic data for the study groups are displayed in table 1 . The mean birth weight in the babies born to pre-eclamptic mothers was noted to be significantly lower than either that of babies born to normotensive women or to women with non-proteinuric pregnancy induced hypertension (table $1 ; \mathrm{p}<0.05$ ). On comparison of the corrected data for gestation for the Nottingham population, only one of the 
babies born to mothers with non-proteinuric pregnancy induced hypertension and four of the babies born to pre-eclamptic mothers were below the 10th centile for birth weight. Only one of the babies ( 29 weeks) born to gestational hypertensive primigravidae died in the early neonatal period, of grade IV intraventricular haemorrhages and respiratory distress syndrome. It is of interest to note the preponderance of male neonates born to primigravidae whose pregnancies were complicated by gestational hypertension. These mothers had a similar proportion of their neonates delivered by caesarean section compared with the normotensive primagravidae $(13 \% v 11 \%)$, in spite of the high risk status of these pregnancies.

(B) BASAL $\mathrm{p}\left[\mathrm{Ca}^{2+}\right] \mathrm{i}$

The mean $\mathrm{p}\left[\mathrm{Ca}^{2+}\right] \mathrm{i}$ concentration was slightly lower for babies born in normotensive primigravidae than non-pregnant subjects. This did not, however, reach statistical significance $(88.9(2.5)$ in normotensive primagravidae $v 93.2(3.6) \mathrm{nmol} / \mathrm{l}$ in nonpregnant volunteers; $p>0 \cdot 1)$. Paired analysis of the umbilical venous and maternal venous $\mathrm{p}\left[\mathrm{Ca}^{2+}\right] \mathrm{i}$ in normotensive primigravidae demonstrated a significant difference (88.9 (2.5) $v$ 119.9 (4.14) $\mathrm{nmol} / \mathrm{l}$; mean (SE) difference $31.06(3.84) ; p<0.0001)$. However, when the umbilical cord $\mathrm{p}\left[\mathrm{Ca}^{2+}\right] \mathrm{i}$ was similarly compared with the $\mathrm{p}\left[\mathrm{Ca}^{2+}\right] \mathrm{i}$ of their mothers at 12 weeks after birth no significant difference was found (mean (SE) difference $-4 \cdot 0(2 \cdot 9)$; table 2$)$.

There was no significant difference between the neonatal cord $\mathrm{p}\left[\mathrm{Ca}^{2+}\right] \mathrm{i}$ in babies born to normotensive primigravidae or those whose pregnancies were complicated by gestational hypertension $(88.9(2.5)$ in normotensive primagravidae; $80.6(2.8)$ in pregnancy induced hypertension without proteinuria, and $89.3(3.2) \mathrm{nmol} / 1$ in pre-eclampsia; ANOVA $p>0 \cdot 19)$. There was also no significant difference in the $\mathrm{p}\left[\mathrm{Ca}^{2+}\right] \mathrm{i}$ of the umbilical cords of the pregnancies studied and those of non-pregnant female volunteers in the follicular phase of their menstrual cycle $(p>0 \cdot 17)$. This was despite a gradual and significant rise in $\mathrm{p}\left[\mathrm{Ca}^{2+}\right] \mathrm{i}$ with increasing severity of disease in the mothers of the babies studied (119 (4.1) in normotensive primagravidae; $130.8(7 \cdot 3)$ in pregnancy induced hypertension without proteinuria, and $148 \cdot 2(4.5) \mathrm{nmol} / 1$ in pre-eclampsia; ANOVA $p<0.025)$. The difference between late intrapartum and umbilical $\mathrm{p}\left[\mathrm{Ca}^{2+}\right] \mathrm{i}$ is thus greater with worsening severity of gestational hypertension (table 2). The mean maternal $\mathrm{p}\left[\mathrm{Ca}^{2+}\right] \mathrm{i}$ in the three samples returned to concentrations comparable with those non-pregnant subjects by 12 weeks after birth and individual data are demonstrated in table 2 .

(C) WHOLE BLOOD PLATELET COUNTS

The maternal whole blood platelet count showed a decrease with worsening severity
Table 3 Total and albumin adjusted calcium concentrations in paired umbilical cord and maternal venous blood in different groups studied; values are mean (SE)

\begin{tabular}{|c|c|c|}
\hline & $\begin{array}{l}\text { Total calcium } \\
(\mathrm{mmol} /)\end{array}$ & $\begin{array}{l}\text { Albumin adjusted } \\
\text { calcium (mmoll) }\end{array}$ \\
\hline \multicolumn{3}{|c|}{ Normotensive primagravidae } \\
\hline $\begin{array}{l}\text { Umbilical } \\
\text { Maternal }\end{array}$ & $\begin{array}{l}2.58(0.06)^{\star \star \star} \\
2.22(0.03)\end{array}$ & $\begin{array}{l}2.62(0.06)^{\star \star \star} \\
2.26(0.02)\end{array}$ \\
\hline \multicolumn{3}{|l|}{ Pregnancy induced } \\
\hline Umbilical & $2.54(0.17)^{\star \star \star}$ & $\begin{array}{l}2.61(0.18)^{\star \star \star} \\
2.28(0.07)\end{array}$ \\
\hline Maternal & $2.24(0.06)$ & $2.28(0.07)$ \\
\hline \multicolumn{3}{|l|}{ Pre-eclampsia } \\
\hline $\begin{array}{l}\text { Umbilical } \\
\text { Maternal }\end{array}$ & $\begin{array}{l}2.47(0.06)^{\star} \\
2.25(0.05)\end{array}$ & $\begin{array}{l}2.25(0.06)^{\star \star} \\
2.36(0.04)\end{array}$ \\
\hline Non-pregnant volunteers & $2.50(0.09)$ & $2.57(0.17)$ \\
\hline
\end{tabular}

${ }^{\star} \mathrm{p}<0.01 ;{ }^{\star \star} \mathrm{p}<0.001 ;{ }^{\star \star \star} \mathrm{p}<0.0001$.

Both the total and albumin corrected serum calcium concentrations were significantly higher in the umbilical cord samples compared with their mothers in the sample groups studied at the time of delivery (non-pregnant volunteers and pregnancy induced hypertension without proteinuria, $\star \star \star p<0.0001 ;$ pre-eclampsia ${ }^{\star \star} p<0.001$ (adjusted) ${ }^{\star} \mathrm{p}<0.01$ (total)).

of gestational hypertension and reached a statistically significant fall in the pre-eclamptic mothers $(245.5(13.6)$ in normotensive primagravidae; $250.5(23.9)$ in pregnancy induced hypertension without proteinuria, and 191.4 (18.5) $\times 10^{9} / 1$ in pre-eclampsia; ANOVA $\mathrm{p}<0.01)$. Although there was a small but consistent fall in platelet count from the umbilical venous samples of the groups studied with worsening gestational hypertension, this did not reach statistical significance $(319.6(16.9)$ in normotensive primagravidae, $296.0(10 \cdot 3)$ in pregnancy induced hypertension without proteinuria, and $264.9(15.7) \times 10^{9} / 1$ in pre-eclampsia; ANOVA $\mathrm{p}>0 \cdot 10$ ).

\section{(D) BIOCHEMISTRY}

There was no significant difference in the mean serum total or albumin adjusted calcium concentration in any of the umbilical blood samples taken in any of the groups studied. However, the albumin adjusted serum calcium concentrations in the umbilical cord blood of all groups, normotensive and hypertensive, were significantly raised compared with paired maternal serum (table 3 ). There was no significant correlation between $\mathrm{p}\left[\mathrm{Ca}^{2+}\right] \mathrm{i}$ and either total or albumin adjusted calcium concentration in either adults or their neonates $(r=0 \cdot 1, \mathrm{p}>0 \cdot 1)$.

\section{Discussion}

Platelets have been identified in the embryonic blood and begin to demonstrate a proaggregatory response by the mid-second trimester. ${ }^{12}$ Platelets are capable of rapid response to a series of physiologically occurring molecules which exert their effects by receptor occupancy. There follows transduction of the surface signal into an intracellular response controlled at least in part by second messengers. ${ }^{1}$ The role of cytosolic free calcium in initiating platelet activity has been shown by elegant experiments demonstrating varying thresholds of cytosolic calcium increase in relation to the platelet response. ${ }^{13}$ The basal $\mathrm{p}\left[\mathrm{Ca}^{2+}\right] \mathrm{i}$ in 'resting' cells, including platelets, 
is approximately $100 \mathrm{nmol} /{ }^{14}$ This concentration is kept by homoeostatic mechanisms that control calcium influx through plasmalemmal channels, the mobilisation of intracellular calcium stores and the $\mathrm{Ca}^{2+}$-ATP-ase pump.

Neonatal platelets seem to be characterised by in vitro functional changes making them less reactive than those present in adults. ${ }^{15}$ It has been noted by Hrodek and Rabiner that neonatal platelets showed decreased adhesion to glass fibres, hypoaggregability to agonists (adrenaline and $\mathrm{ADP}$ ), and a decreased platelet factor III activity. ${ }^{16} \mathrm{~A}$ subsequent study by Mull and Hathaway investigating platelet aggregation in 60 neonates demonstrated impaired aggregation in the presence of ADP, collagen, and thrombin compared with adults. ${ }^{3}$ It was concluded by the investigators that these data could not be explained by the presence of either a plasma inhibitor or by the platelets being in a refractory state secondary to increased circulating concentrations of ADP. It was thought to be an inherent property of the platelets themselves. Others measuring the percentage aggregation in platelet rich plasma obtained from umbilical cord blood have only been able to confirm a reduced percentage aggregation induced by low concentrations of ADP $(2 \mu \mathrm{M})$, collagen, and adrenaline. ${ }^{4}$ The use of whole blood platelet aggregation and exocytosis using proaggregatory agents has confirmed these data using this more physiological ex vivo technique. ${ }^{6}$ The lesser aggregatory response of neonatal platelets to adrenaline might be explained by a reduced number of $\alpha_{2}$-adrenoreceptors on these platelets or it may be that these receptors are in some way 'down regulated'. ${ }^{17}$ The platelets obtained from neonates seem to have a reduced 'storage system' that has a corresponding effect on the ability of these cells to undergo exocytosis. This change is either because of a decreased uptake of the precursors of amine synthesis and concentrating ability within these granules or because of a defect in retention. This is particularly noticeable in the low endogenous concentrations of serotonin. ${ }^{18}$ Further evidence of dense body content depletion is provided by Suarez et $a l,{ }^{19}$ who noted that serum cord blood $\beta$-thromboglobulin concentrations are raised at delivery.

These data demonstrate no significant difference between the mean $\mathrm{p}\left[\mathrm{Ca}^{2+}\right] \mathrm{i}$ in non-pregnant women and those obtained from the umbilical venous blood of normotensive primigravidae. They suggest that the functional hypoactivity of neonatal platelets is probably not secondary to a decrease in basal $\mathrm{p}\left[\mathrm{Ca}^{2+}\right] \mathrm{i}$. However, the mean $\mathrm{p}\left[\mathrm{Ca}^{2+}\right] \mathrm{i}$ of neonatal platelets was consistently lower than for non-pregnant adults. Although there is a substantial degree of overlap between the groups, the small sample size means that the possibility of a type II error cannot be completely excluded. Likewise, unpaired analysis demonstrated no significant difference between the neonatal $\mathrm{p}\left[\mathrm{Ca}^{2+}\right] \mathrm{i}$ of babies born to normotensive primigravidae and those pregnancies complicated by gestational hypertension. It may be that removing the platelets from the other constituent parts of whole blood alters the behaviour of the cells. This is supported by previous evidence suggesting that arachidonate stimulated production of thromboxane is increased in neonatal platelets compared with adults. This difference was lost when the platelets were prepared as a washed suspension. ${ }^{20}$ This implies that a possible plasma factor that is transferable may be involved. A difference was demonstrable on paired analysis of the neonatal and maternal $\mathrm{p}\left[\mathrm{Ca}^{2+}\right] \mathrm{i}$ in the immediate postpartum period, in both normotensive and hypertensive pregnancy. This difference disappeared by 12 weeks after birth, when the majority of evidence indicates that the influence of pregnancy on platelet behaviour has ceased. ${ }^{6}$ This in itself is an interesting observation as it implies that the 'factor' raising the basal $\mathrm{p}\left[\mathrm{Ca}^{2+}\right] \mathrm{i}$ in the mother is either outside the sphere of influence of the fetoplacental unit or the fetal/neonatal platelets are resistant to its effects. One may speculate that the placenta itself may form a protective barrier or that the change occurs at the receptor level on the platelet membrane and a degree of receptor resistance is present. It is also possible that the second messenger system which predominates in these immature platelets is different from that present in adults. Cyclic adenosine monophosphate (cAMP) is the intracellular second messenger that has a predominately inhibitory effect. It may be that modification of the adenyl cyclase system in the neonatal platelets is responsible for their observed in vitro hypoactivity and that $\mathrm{p}\left[\mathrm{Ca}^{2+}\right] \mathrm{i}$ has no controlling role in such circumstances. Such comparative data have not been published at the present time.

Calcium homoeostasis within a cell is determined by the flow of calcium ions from the extracellular fluid through 'channels', the release of calcium from intracellular stores and by active pumping of calcium out of the cell. These data also demonstrate no significant correlation between either $\mathrm{p}\left[\mathrm{Ca}^{2+}\right] \mathrm{i}$ and serum calcium concentrations in either neonates or their mothers in the groups studied. To what extent any of these factors influence the cytosolic calcium concentration in the newborn is unknown.

This work was sponsored by Birthright.

1 Haslam RJ. Signal transduction in platelet activation. State of the art lecture. XIth Congress Haemostasis and of the art lecture. XIth Congress Haemo

2 Rink TJ. Cytosolic calcium in platelet activation. Experientia 1988; 44: 97-100.

3 Mull MM, Hathaway WE. Altered platelet function in the newborn. Pediatr Res 1978; 4: 229-37.

4 Corby DG, Zuck TF. Newborn platelet dysfunction: a storage pool and release defect. Thromb Haemost 1976; 36: $200-7$.

5 Stuart MJ. The neonatal platelet: evaluation of the platelet malionyldialdehyde formation as an indicator of prostaglandin synthesis. Br f Haematol 1978; 39: 83-90.

6 Louden KA, Broughton Pipkin F, Heptinstall S, et al. Neonatal platelet reactivity and serum $\mathrm{TXB}_{2}$ production in whole blood: the effects of maternal low dose aspirin. in whole blood: the effects of maternal

7 Kilby MD, Broughton Pipkin F, Symonds EM. Calcium and platelets in human pregnancy. $\mathcal{F}$ Hypertens 1992; 10: a97-1003. 
8 MacGillivray I, Davey DA. Classification of hypertensive disorders of pregnancy. In: Sharp F, Symonds EM, eds. Proceedings of the 16th study group of RCOG. Ithaca, NY: Perinatology Press, 1986: 401-8.

9 Hallam TJ, Sanchez A, Rink TJ. Stimulus-response coupling in human platelets. Biochem 7 1984; 218: couplin

10 Kilby MD, Cockbill SC, Broughton Pipkin F, Heptinstall S, Symonds EM. A cross sectional study investigating platelet intracellular free calcium in normotensive
hypertensive pregnancies. Clin Sci 1990; 78: 75-80.

1 Statistical package for the social sciences. Version 6.0 for Windows. New York: McGraw Hill, 1993.

12 Pandolfi M, Àstedt B, Cronberg I, Nilsson IM. Failure of fetal platelets to aggregate in response to adrenaline and collagen. Proc Soc Exp Biol Med 1972; 141: 1081-3.

13 Rink TJ, Hallam T. What turns platelets on? Trends Biochem Sci 1984; 9: 215-9.

14 Rink TJ, Smith SW, Tsien RY. Cytosolic calcium in human platelets: calcium thresholds and independent activation
for shape change and secretion. FEBS Lett 1982; 148: for shape
$21-6$.
15 Whaun JM. Platelet function in the neonate. In: Stockman JA III, Pechedly C, eds. Developmental and neonatal haematology. New York: Raven Press, 1988: 265-81.

16 Hrodek O, Rabiner FS. Abnormalities of platelet factor 3 availability and content in newborn infants. In: Deutsch $\mathrm{E}$, Gerlach E, Moser K, eds. Permeability of erythrocytes and thrombocytes. Stuttgart: Schanter-Verlag, 1968 : 293-5.

17 Corby DG, Barber JA, O'Barr TP. Decrease in $\alpha_{2}$-adrenergic receptor binding sites in newborn platelets. A cause of abnormal response to adrenaline. Dev Pharmolcol Ther $1981 ; 2: 215-25$.

18 Whaun JM. The platelets of the newborn infant: 5-HT uptake and 'release'. Thrombosis et Diathesis Haemorrhagica 1973; 30: 327-33.

19 Suarez CR, Menendez CE, Walgena RW, Fareed J. Neonatal and maternal haemostasis. Semin Thromb Hemost 1984; 10: 280-4.

20 Stuart MJ, Dusse J, Clarke DA, Walgena RW. Differences in thromboxane production between neonatal and adult in thromboxane production between neonatal and adult Pediatr Res 1984; 18: 823-6. 